\title{
Anti-Cytokeratin CAM 5.2 (Becton Dickinson) is not synonymous with CK8/18 monoclonal antibody. Comment on "Pancreatic-type mixed acinar-endocrine carcinoma with alpha-fetoprotein production arising from the stomach: a report of an extremely rare case. Med Mol Morphol (2009) 42:167-174"
}

Received: November 18, 2009 / Accepted: January 7, 2010

Key words Anti-cytokeratin · Cytokeratin · CAM 52 - CK8/ $18 \cdot$ Synonymous

To the Editor:

In the recent report of one interesting case, entitled "Pancreatic-type mixed acinar-endocrine carcinoma with alpha-fetoprotein production arising from the stomach: a report of an extremely rare case," in Medical Molecular Morphology (2009) 42:167-174, we found that the authors may have inadvertently annotated, in Table 1 of that article, that the antibody to $\mathrm{CK} 8 / 18$ was derived from clone "CAM5.2," Becton Dickinson (BD), San Jose, CA, USA [1].

In 1997, Becton Dickinson Biosciences (San Jose, CA, USA) revised the data sheet for CAM 5.2, which gave the primary reactivity as cytokeratin 8 and, to a lesser extent, for the closely related cytokeratin 7 . On the other hand, it shows no reactivity with 18 or 19 [2,3]. We would like to clarify that CAM 5.2 (Becton Dickinson) does not stand for
CK 8/18. This letter, hereby, can help us elucidate the point that anticytokeratin CAM 5.2 (B.D.) is not synonymous with the cytokeratin $8 / 18$ monoclonal antibody.

\section{References}

1. Kusafuka K, Bando E, Muramatsu K, Ito H, Tanizawa Y, Kawamura T, Mochizuki T, Terashima M, Nakajima T (2009) Pancreatic-type mixed acinar-endocrine carcinoma with alpha-fetoprotein production arising from the stomach: a report of an extremely rare case. Med Mol Morphol 42:167-174

2. Becton Dickinson (1997) Anti-Cytokeratin (CAM 5.2) Reagent for in vitro diagnostic use 50 tests per vial. Catalog no. 349205, 9/97, 23-3190-01, and Material Safety Data Sheet. Accessed to ISO/DIS 11014. http://www.bdbiosciences.com/ptProduct.jsp?prodId $=22700$ and http://www.bdbiosciences.com/external_files/is/doc/msds/live/ web_enabled/349205-MSDS-USEN.A.pdf (cited Jan 7, 2010)

3. Han CP, Hsu JD, Lee MY, Kok LF, Wang PH, Tyan YS, Yao CC (2009) True cytokeratin $8 / 18$ immunohistochemistry is of no use in distinguishing between primary endocervical and endometrial adenocarcinomas in a tissue microarray study. Int $\mathrm{J}$ Gynecol Pathol (2010, in press)
J.-D. Hsu

Department of Pathology, Chung Shan Medical University Hospital, Taichung, Taiwan

J.-D. Hsu

Department of Pathology, School of Medicine, Chung Shan Medical University, Taichung, Taiwan

C.-P. Han $(\bowtie)$

Department of Obstetrics and Gynecology, Chung-Shan Medical

University Hospital, No. 110, Sec. 1, Chien-Kuo N. Road Taichung,

Taiwan, ROC

Tel. +886-4-24739595 Fax +886-4-35073516

e-mail: hanhaly@gmail.com

C.-P. Han

Institute of Medicine, Chung-Shan Medical University, Taichung,

Taiwan, ROC

C.-P. Han

Clinical Trial Center, Chung-Shan Medical University Hospital,

Taichung, Taiwan, ROC 